\title{
Single megadose vitamin A supplementation of Indian mothers and morbidity in breastfed young infants
}

\author{
S Basu, B Sengupta, P K Roy Paladhi
}

Postgrad Med J 2003;79:397-402

See end of article for authors' affiliations

Correspondence to: Dr Sriparna Basu, 113 Ultadanga Main Road Calcutta 700 067, India; drsriparnabasu@ rediffmail.com

Submitted 25 September 2002

Accepted

29 January 2003

\begin{abstract}
Background and objective: In developing countries low maternal vitamin A stores combined with increased demands of pregnancy and lactation may lead to its deficiency in breastfed infants. This study evaluates the effects of maternal supplementation with a high dose of vitamin $A$ on the serum retinol levels of exclusively breastfed infants, and their morbidity in the first six months of life.

Setting: Hospital based.

Study design: Randomised controlled trial.

Subjects and methods: Mothers of the test group $(n=150)$ were orally supplemented with a single dose of retinol (209 $\mu \mathrm{mol})$ soon after delivery and were advised exclusive breastfeeding for six months. Before supplementation retinol levels were estimated in the mothers' and newborns' blood, and in colostrum. On follow up, breastmilk and infants' serum retinol contents were assessed monthly for six months. Retinol level $<0.7 \mathrm{mmol} / \mathrm{l}$ indicated vitamin A deficiency. Morbidity patterns like vitamin A deficiency, diarrhoea, febrile illnesses, acute respiratory infection, measles, and ear infection were also studied and compared between the two groups.

Results: Presupplement mean maternal serum retinol levels were 0.98 and $0.92 \mu \mathrm{mol} / \mathrm{l}$ and mean breastmilk levels were 3.85 and $3.92 \mu \mathrm{mol} / /$ in the test and control groups respectively $(p>0.05)$. Mean cord blood retinol levels were also comparable $(0.68 \vee 0.64 \mu \mathrm{mol} / \mathrm{l})$. After supplementation, the test group showed a rise in mean breastmilk retinol content $(12.08 \vee 2.96 \mu \mathrm{mol} / \mathrm{l})$ which remained significantly higher for four months. The infants' mean serum retinol level, initially $322.06 \%$ of the baseline value, was significantly higher for five months. In the control group, significant numbers of mothers and infants showed deficient breastmilk and serum retinol throughout the follow up $(p<0.01)$. Decreased incidence and duration of various diseases were also found in the test group suggesting lesser morbidity.

Conclusion: Maternal supplementation with single megadose vitamin A is an effective strategy for
\end{abstract} vitamin A prophylaxis of exclusively breastfed infants of 0-6 months.
$\mathrm{V}$ itamin A supplementation and food fortification have beneficial effects on infant mortality and morbidity. ${ }^{12}$ The vitamin A status of most newborns is marginal and prevention from its deficiency may be ensured by breastfeeding, provided the retinol concentration in the breastmilk is adequate. ${ }^{3}$ Women in developing countries have an average daily intake of vitamin A of less than half and an average serum retinol concentration that is $70 \%$ of women in developed countries. ${ }^{4}$ In this situation even exclusive breastfeeding may not protect the newborn from vitamin A deficiency (VAD) and may lead to diseases, clinically overt (xerophthalmia, anaemia, growth retardation, increased infectious morbidity, and mortality) or occult (impaired iron metabolism, poor cellular differentiation, and depressed immune response). ${ }^{5-7}$ These result in increased rate and complications of respiratory tract infections, diarrhoeal diseases, and further micronutrient deficiency. ${ }^{8-10}$ VAD is also associated with increased incidence of measles. ${ }^{11}{ }^{12}$ The anaemia of VAD is a subject of interest in the present day. Its pathogenesis is through diverse biological mechanisms such as effect on growth and differentiation of erythrocyte progenitor cells, immunity to infections, and mobilisation of iron stores from the tissues. ${ }^{13}$

India's national programme on VAD diseases ensures vitamin A supplementation of infants from the age of 6 months. ${ }^{14}$ Hence in the first six months of life the infant may remain unprotected if the vitamin A content of the mother's breastmilk is low. However, there is a growing awareness that subclinical or overt deficiency can occur in infants less than 6 months of age. ${ }^{15}$ This study attempts to evaluate the effect of a single oral megadose of vitamin A on the breastmilk concentration, the infants' serum level, and infant morbidity patterns during the first six months of life in an urban population of West Bengal, India.

\section{SUBJECTS AND METHODS}

A randomised controlled prospective study was conducted in the Department of Paediatrics, NRS Medical College and Hospital, Calcutta, India. It was approved by the hospital ethical committee. Apparently healthy pregnant women without any history of hypertension, diabetes, or any other chronic medical illness were recruited consecutively in their third trimester (beyond 34 weeks) from the antenatal clinic of the hospital. Inclusion criteria were at least two completed antenatal visits, tetanus immunisation in pregnancy, and term vaginal delivery. Those with stillbirth, an eventful peripartum period, or who received vitamin A supplementation in pregnancy were excluded. A total of 300 such mothers were finally recruited to the study; informed consent was received from all participants in the trial. The mothers along with their babies were randomised by systematic sampling technique to test and control groups of 150 each (alternate allocation to the test and control group). All were questioned about their detailed dietary habits from which an approximate daily intake of vitamin A was calculated by using the Indian food table. ${ }^{16}$ The newborns' birth weights were recorded and the gestational ages were assessed using the Dubowitz criteria. ${ }^{17}$

For estimation of serum retinol, 3-5 $\mathrm{ml}$ of cord blood from the newborns and 3-5 $\mathrm{ml}$ of venous blood from the mothers 
300 mother-newborn pairs

Inclusion criteria:

1. At least 2 antenatal visits

2. Tetanus immunisation in

pregnancy

3. Term vaginal delivery

4. Informed consent

Exclusion criteria:

1. Stillbirth

2. Eventful peripartum period

3. Vitamin A supplementation in pregnancy

4. Chronic illnesses in the mother

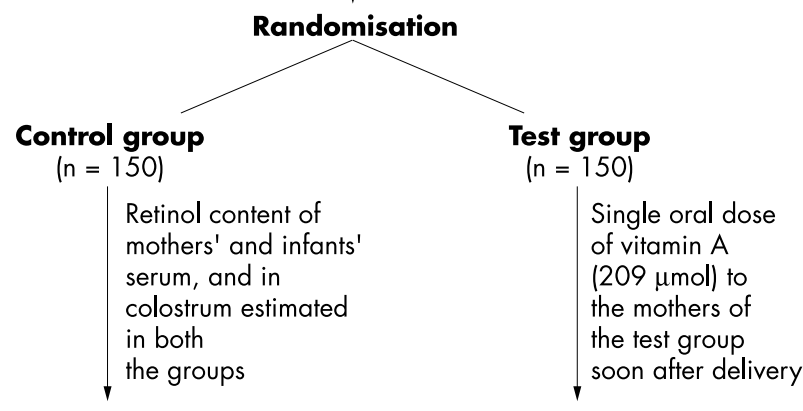

- Exclusive breastfeeding for 6 months

- Follow up monthly for 6 months

- Any illness in the babies, report immediately At each follow up:

- Estimate retinol level in breast milk and infants' serum

- Examine for any sign of VAD

- Record the total number of episodes of diarrhoea, ARI, measles, febrile illness, and ear infection, and compare between the two groups

- Examine the baby thoroughly

$$
\begin{aligned}
& \text { - Any sign of VAD, withdraw } \\
& \text { the child from the study and } \\
& \text { supplement adequately } \\
& \text { - Treat infections accordingly }
\end{aligned}
$$

Calculate the mean (SD) and $95 \%$ confidence intervals Analyse data by the $Z$ test; $p<0.05$ = significant

Figure 1 Protocol used for the study.

were collected, the serum separated, and transferred to stopped tubes. Colostrum was collected by manual breast pumps. Samples from both the breasts were mixed and $5 \mathrm{ml}$ of this mixed sample was collected in a stopped tube. Both the serum and milk samples were protected from light, placed in a cool box at $4-8^{\circ} \mathrm{C}$, and transferred within four hours to the central laboratory. Retinol concentrations were estimated by the modified method of Neeld and Pearson. ${ }^{18}$

All neonates were examined thoroughly. A single oral dose of $209 \mu \mathrm{mol}$ of retinol (200 $000 \mathrm{IU}$ of vitamin A) was given to each mother of the test group only within 24 hours of delivery and after the blood and breastmilk samples were collected. No prelacteal feeds were given to the neonates and all the mothers were strongly motivated for exclusive breastfeeding for six months. The process of motivation was initiated during the antenatal period and when they were admitted for childbirth. They were discharged from the hospital only after establishment of exclusive breastfeeding. Constant motivation for exclusive breastfeeding continued at all points of contact.

These mother-child pairs were called for monthly follow up for six months. However those who did not exclusively breastfeed their infants were not excluded from the study and were followed up like others for any sign of VAD. This was done on an "intention to treat" basis. In case of any illness of the infants during this period, mothers were instructed to contact the first author either in the paediatric outpatient department or in the ward and the nature, duration, and number of

\section{Box 1: Definition of illness in infants}

- Episode of diarrhoea: $\geqslant 1$ day in which there are $\geqslant 3$ stools of watery and loose consistency or any loose stool with blood.

- Episode of upper respiratory infection: $\geqslant 1$ day of cough or nasal discharge or purulent ear drainage.

- Episode of lower respiratory infection: $\geqslant 1$ day of combination of cough with indrawing of the lungs. Three or more symptom-free days differentiate episodes of diarrhoea or respiratory tract infections.

- Measles: Clinical signs of maculopapular skin rash with its typical distribution and fever with cough, coryza, or conjunctivitis was defined as measles.

- Fever: Rise of body temperature above $39^{\circ} \mathrm{C}$ axillary for a period of 48 hours or more was defined as fever.

- Vitamin A deficiency (VAD): VAD in mothers was considered with the presence of night blindness, Bitot's spot, corneal xerosis, or corneal ulcer. In infants up to 9 months, conjunctival xerosis, Bitot's spot, or corneal ulcers were considered as VAD.

- Ear infection: (A) Acute infection (serous/suppurative) was characterised by the presence of earache (or persistent crying on tugging at the ears) and examination with the otoscope reveals a hyperaemic, opaque bulging tympanic membrane or purulent discharge. (B) Chronic infection (serous/mucoid/purulent) was noted if there were symptoms of dull pain for a long period, fever with purulent discharging ears, or otoscopic examination reveals perforation of tympanic membrane with or without purulent discharge.

episodes of such diseases were recorded. At each follow up 3-5 $\mathrm{ml}$ of venous blood from the infants and $5 \mathrm{ml}$ of breastmilk were collected from both the groups. Mothers were instructed not to breastfeed their infants after early morning on the follow up days. Breastmilk samples were collected between 9 am and $11 \mathrm{am}$. Method of sample collection, transport, and retinol estimation were the same as described above. The infants were thoroughly examined at each visit. The total number of episodes and duration of diarrhoeal disease, acute respiratory infection, febrile illness, measles, and ear infection of the infants in the last one month were asked about and recorded. For defining and diagnosing morbidity the disease definitions of Rahmathulla et al (box 1) were followed. ${ }^{19}$ Lower respiratory infections and upper respiratory infections were both considered as acute respiratory infections. The infants who developed signs of VAD including those not exclusively breastfed were omitted from the study at the respective point of time and treated accordingly. The mean (SD) and 95\% confidence interval of birth weights, maternal age, body weight, dietary intake of retinol, serum and breastmilk retinol levels (predose and at each follow up), and morbidity data were calculated in both the groups. Statistical significance was assessed by the $\mathrm{Z}$ test and $\mathrm{p}$ value less than 0.05 was taken as statistically significant.

The flow diagram (fig l) sums up the protocol used for the study.

\section{RESULTS}

Both the groups were comparable with respect to maternal age and body weight, birth weight and gestational age of the newborns, and mean maternal dietary intake of retinol (table 1). The presupplementation mean maternal serum retinol levels were $0.98(0.93-1.03) \mu \mathrm{mol} / \mathrm{l}$ and $0.92(0.86-0.98) \mu \mathrm{mol} / \mathrm{l}$ and the presupplementation mean breastmilk retinol levels were $3.85(3.65-4.05) \mu \mathrm{mol} / \mathrm{l}$ and $3.92(3.69-4.15) \mu \mathrm{mol} / \mathrm{l}$ in the test and control group respectively without any significant difference (table 2).

Out of 150 mothers in either group, 139 in the test and 132 in the control completed the six months' follow up and exclusively breastfed their babies. Four in the test and 10 in the 
Table 1 Sample characteristics of the tests and the control groups; results are mean ( $95 \%$ confidence interval)

\begin{tabular}{|c|c|c|}
\hline & Test group & Control group \\
\hline Maternal age (years) & 25.2 (24.5 to 25.8$)$ & $24.6(23.9 \text { to } 25.2)^{*}$ \\
\hline Maternal body weight $(\mathrm{kg})$ & 43.3 (42.5 to 44.1$)$ & $44.2(43.2 \text { to } 45.2)^{*}$ \\
\hline Birth weight of newborn $(\mathrm{kg})$ & $2.7(2.5$ to 2.9$)$ & $2.6(2.45 \text { to } 2.75)^{*}$ \\
\hline Gestational age of newborns (weeks) & $39.4(39.1$ to 39.7$)$ & $39.0(38.3 \text { to } 39.7)^{*}$ \\
\hline Maternal average daily intake of retinol ( $\mu \mathrm{mol})$ & 48.0 (46.8 to 49.2$)$ & $47.0(45.7 \text { to } 48.3)^{*}$ \\
\hline Presupplementation maternal serum retinol level $(\mu \mathrm{mol} / \mathrm{l})$ & $0.98(0.93$ to 1.03$)$ & $0.92(0.86 \text { to } 0.98)^{*}$ \\
\hline Presupplementation breastmilk retinol level $(\mu \mathrm{mol} / \mathrm{I})$ & 3.85 (3.65 to 4.05$)$ & $3.92(3.69 \text { to } 4.15)^{*}$ \\
\hline
\end{tabular}

Table 2 Breastmilk retinol levels ( $\mu \mathrm{mol} / \mathrm{l})$ after supplementation and on follow up; results are mean $(95 \%$ confidence interval)

\begin{tabular}{lll}
\hline & Test group & Control group \\
\hline 24 hours after supplementation & $12.08(11.45$ to 12.71$), \mathrm{n}=150$ & $2.96(2.74 \text { to } 3.18)^{*}, \mathrm{n}=150$ \\
Follow up (months) & $1.90(1.79$ to 2.01$), \mathrm{n}=150$ & $1.08(1.00 \text { to } 1.16)^{*}, \mathrm{n}=149$ \\
1 & $1.74(1.66$ to 1.82$), \mathrm{n}=149$ & $0.90(0.80 \text { to } 1.00)^{*}, \mathrm{n}=148$ \\
2 & $1.54(1.46$ to 1.62$), \mathrm{n}=149$ & $0.81(0.73 \text { to } 0.89)^{*}, \mathrm{n}=145$ \\
3 & $1.20(1.11$ to 1.29$), \mathrm{n}=146$ & $0.73(0.66 \text { to } 0.80)^{*}, \mathrm{n}=141$ \\
4 & $0.72(0.62$ to 0.82$), \mathrm{n}=146$ & $0.70(0.63$ to 0.77$), \mathrm{n}=140$ \\
5 & $0.71(0.63$ to 0.79$), \mathrm{n}=146$ & $0.67(0.59$ to 0.75$), \mathrm{n}=140$ \\
6 & &
\end{tabular}

Table 3 Serum retinol levels ( $\mu \mathrm{mol} / \mathrm{l}$ ) of infants at delivery and on follow up

\begin{tabular}{|c|c|c|c|c|}
\hline & \multicolumn{2}{|l|}{ Test group } & \multicolumn{2}{|l|}{ Control group } \\
\hline & $\begin{array}{l}\text { Mean ( } 95 \% \text { confidence } \\
\text { interval) }\end{array}$ & $\begin{array}{l}\text { Increase above } \\
\text { baseline value (\%) }\end{array}$ & $\begin{array}{l}\text { Mean }(95 \% \text { confidence } \\
\text { interval) }\end{array}$ & $\begin{array}{l}\text { Increase above } \\
\text { baseline value (\%) }\end{array}$ \\
\hline $\begin{array}{l}\text { At delivery (presupplementation } \\
\text { baseline value) } \\
\text { Follow up (months) }\end{array}$ & $0.68(0.64$ to 0.72$), n=150$ & - & $0.64(0.60$ to 0.68$), n=150$ & - \\
\hline 1 & $2.87(2.79$ to 2.88$), n=150$ & 322.06 & $0.92(0.84 \text { to } 1.00)^{*}, n=149$ & 43.75 \\
\hline 2 & $2.17(2.06$ to 2.28$), n=149$ & 219.12 & $0.85(0.77 \text { to } 0.93)^{*}, \mathrm{n}=148$ & 32.81 \\
\hline 3 & 1.60 (1.52 to 1.68$), n=149$ & 135.29 & 0.80 (0.73 to 0.87$)^{*}, n=144$ & 25.00 \\
\hline 4 & $1.41(1.31$ to 1.51$), \mathrm{n}=146$ & 107.35 & $0.72(0.62 \text { to } 0.82)^{*}, n=137$ & 12.50 \\
\hline 5 & $1.06(0.98$ to 1.14$), n=146$ & 55.88 & $0.70(0.61 \text { to } 0.79)^{*}, \mathrm{n}=134$ & 9.38 \\
\hline 6 & $0.72(0.62$ to 0.82$), n=146$ & 5.38 & 0.68 (0.58 to 0.88$), n=134$ & 6.25 \\
\hline
\end{tabular}

control group dropped out and were excluded from the study. Seven in the test and eight in the control did not exclusively breastfeed their babies. The breastmilk retinol concentration increased significantly in the test population within 24 hours of supplementation. On follow up, a gradual decline in the breastmilk retinol levels was observed in both the groups. However, the test population showed a significantly higher level up until four months of follow up (table 2). Before supplementation, cord blood retinol levels were comparable in both the groups. On follow up the serum retinol levels of the infants in the test population remained significantly higher until five months of life (table 3 ).

Before supplementation, the percentage of mothers below the cut off for deficient serum retinol were comparable in both the groups $(34.66 \% \vee 32.67 \%)$. At birth, $48 \%$ and $52 \%$ of infants were below the cut off for deficient serum retinol in the test and control groups respectively (table 4 ). Though the number of subjects below the cut off for both breastmilk and infant's serum retinol was significantly lower in the test group throughout the follow up, a progressive increase in the number was seen after the third month (table 4).

Significant differences were seen in the number of episodes and duration of diarrhoea and acute respiratory infection between the two groups (table 5). Infants of the supplemented mothers had fewer episodes of febrile illness. One infant in the test group and seven in the control group had a measles infection. None of the infants in the test group, including those not exclusively breastfed, showed any sign of VAD. A total number of six babies (four exclusively breastfed and two not exclusively breastfed) in the control developed VAD-one in the third month, three in the fourth month, and two in the fifth month of follow up. They were omitted from the study at these respective times and adequately supplemented with vitamin A, though their mothers went on to complete the follow up. There was no significant difference in the incidence of ear infection between the two groups (table $5)$.

\section{DISCUSSION}

In the present study preterm infants were excluded as they might have poor storage of vitamin A at birth and hence develop VAD earlier. The study subjects represent a low income section of the urban population and the profile of such a target group in terms of maternal body weight, average daily retinol intake, baseline maternal serum, and breastmilk retinol levels has been taken into consideration. The vitamin $\mathrm{A}$ status of pregnant and lactating women tends to vary in different economic, ethnic, and regional groups. Most workers accept levels less than $0.7 \mu \mathrm{mol} / \mathrm{l}(20 \mu \mathrm{g} / \mathrm{dl})$ as diagnostic of 
Table 4 Comparison of number of subjects (absolute and percentage) below cut off value (0.7 $\mu \mathrm{mol} / \mathrm{l})$ for deficient breastmilk and serum retinol

\begin{tabular}{|c|c|c|c|c|}
\hline & \multicolumn{2}{|c|}{ No (\%) of mothers with deficient breastmilk retinol } & \multicolumn{2}{|c|}{ No (\%) of infants with deficient serum retino } \\
\hline & Test group & Control group & Test group & Control group \\
\hline Presupplementation (mother) & $15(10.0)$ & $12(8.0)$ & - & - \\
\hline At delivery (infant) & - & - & $48(32.0)$ & $52(34.67)$ \\
\hline \multicolumn{5}{|l|}{ Follow up (months) } \\
\hline 1 & $0(0)$ & $14(9.40)^{*}$ & $0(0)$ & $22(14.77)^{*}$ \\
\hline 2 & $0(0)$ & $21(14.19)^{*}$ & $0(0)$ & $34(22.97) *$ \\
\hline 3 & $1(0.67)$ & $32(22.07)^{*}$ & $0(0)$ & $53(36.81)^{*}$ \\
\hline 4 & $4(2.74)$ & $41(29.08)^{*}$ & $2(1.37)$ & $71(51.82)^{*}$ \\
\hline 5 & $12(8.22)$ & $52(37.14)^{*}$ & $19(13.01)$ & $78(58.21)^{*}$ \\
\hline 6 & 19 (13.01) & $71(50.71)^{*}$ & $32(21.92)$ & $92(68.66)^{*}$ \\
\hline
\end{tabular}

Table 5 Comparison of morbidity patterns of the test and control groups during follow up; results are mean $195 \%$ confidence interval)

\begin{tabular}{|c|c|c|c|}
\hline \multirow[b]{2}{*}{ Number of diarrhoeal episodes (cumulative) } & \multicolumn{2}{|c|}{ Test group } & \multirow{2}{*}{$\begin{array}{l}\text { Control group } \\
1.61(1.31 \text { to } 1.64)^{*}, n=240\end{array}$} \\
\hline & 0.10 & $(0.005$ to 0.105$), n=15$ & \\
\hline Diarrhoea duration (days) & 0.96 & (0.91 to 1.01$)$ & $2.38(2.18 \text { to } 2.58)^{*}$ \\
\hline Number of episodes of ARI (cumulative) & 0.34 & $(0.32$ to 0.36$), n=51$ & $2.55(2.35 \text { to } 2.75)^{*}, n=380$ \\
\hline ARI duration (days) & 2.90 & $(2.70$ to 3.10$)$ & $5.61(5.11 \text { to } 6.11)^{*}$ \\
\hline Number of episodes of febrile illness (cumulative) & 0.20 & $(0.192$ to 0.208$), n=30$ & $0.34(0.32 \text { to } 0.36)^{*}, n=51$ \\
\hline Number of infants with measles infection & 0.006 & $(0.005$ to 0.007$), n=1$ & $0.05(0.043 \text { to } 0.057)^{*}, \mathrm{n}=7$ \\
\hline Number of infants with VAD & 0.00 & $(0.00$ to 0.00$), n=0$ & $0.04(0.039 \text { to } 0.041)^{*}, \mathrm{n}=6$ \\
\hline Number of episodes of ear infection (cumulative) & 0.01 & $(0.002$ to 0.018$), n=2$ & $0.01(0.003$ to 0.017$), n=2$ \\
\hline
\end{tabular}

VAD with levels less that $0.35 \mu \mathrm{mol} / \mathrm{l}(10 \mu \mathrm{g} / \mathrm{dl})$ closely correlating with inadequate body stores of vitamin $\mathrm{A}$ and clinical manifestations of VAD. ${ }^{20}{ }^{21}$ Similar values are used for defining deficient breastmilk retinol levels with a baseline deficiency being less than $1.05 \mu \mathrm{mol} / \mathrm{l}(30 \mu \mathrm{g} / \mathrm{dl}){ }^{21}{ }^{21}$ In this study we have taken a retinol level of $0.7 \mu \mathrm{mol} / \mathrm{l}$ as cut off for deficiency in serum and breastmilk. The mean baseline maternal serum retinol was lower than that reported by Roy et al $(0.98 v 1.38$ $\mu \mathrm{mol} / \mathrm{l})$ whereas the mean baseline breastmilk retinol levels were comparable $(3.85 \vee 3.20 \mu \mathrm{mol} / \mathrm{l}){ }^{22} \mathrm{~A}$ high percentage of mothers in both the test and control groups (34.66\% v 32.67\%) were below the cut off level of baseline serum retinol which projects the vitamin A status in the postpartum mothers in this study. Despite such high values few mothers at delivery (before supplementation) had a breastmilk retinol content below the cut off value (10\% and $8 \%$ in the test and control respectively). This is probably due to high levels of vitamin A in colostrum, which is almost uniform in postpartum mothers irrespective of serum retinol levels (table 4). The benefit of immediate postpartum supplementation of mothers with a single megadose was seen in the breastmilk of the test group for up to four months of follow up. The raised serum level found for up to five months of follow up has adequately protected the test group infants from VAD.

A variety of factors like recurrent infections, worm infestations, stress, variable dietary provitamin A intake, and lactational demands tend to affect vitamin A metabolism and stores in the mother and hence the breastmilk levels. Standardisation of all these variables is difficult and beyond the scope of this study. Ideally the hepatic retinol content best depicts the vitamin A status, the estimation of which was not practically feasible. We did not include serial maternal serum retinol estimation, which might reflect the vitamin A utilisation of the mothers after supplementation. Our main aim was to assess the effect of maternal supplementation on infant morbidity. Breastmilk retinol level is a better parameter as it directly attributes the availability of the vitamin to the infant. Moreover, its estimation is non-invasive, readily acceptable, and sample collection is relatively easy.

A number of previous studies have shown the benefits of postpartum vitamin A supplementation to mothers, either direct or through fortified foods. Food based interventions either use fortified foods like sugar, monosodium glutamate, or $\beta$-carotene rich fruits and vegetables. Questions remain on their efficacy and the ultimate aim of improving infant's serum retinol level. Study using fortified monosodium glutamate showed an increase in vitamin A level in the mother but not in the infant. ${ }^{23}$ In another study it was shown that retinol levels in the maternal serum and breastmilk increase after consumption of synthetic supplements of $\beta$-carotene but not $\beta$-carotene in vegetables. ${ }^{24}$ Studies in which direct supplementation was done showed a satisfactory outcome. In a non-randomised study Thanangkul et al demonstrated a rise in maternal serum retinol levels for nine months, breastmilk levels for seven months, and a rise in the infants' serum retinol at 1-7 months postpartum after a single dose of $312 \mu \mathrm{mol}$ vitamin $\mathrm{A}$ to the mothers at delivery. ${ }^{25} \mathrm{~A}$ similar dose had been used by Stoltzfus et al in Indonesian mothers at 1-3 weeks postpartum. ${ }^{26}$ The breastmilk retinol content remained high up to eight months and also benefited the infants. In these studies, the higher dose of vitamin A was probably responsible for the high retinol levels for a longer period. Roy et al used a dose similar to ours and the effect achieved in the infants was for six months, the usual recommended period of breastfeeding. ${ }^{22}$ Vinutha et al used a similar dose but their follow up was for three months only. ${ }^{21}$

Recently, in a multicentre trial, Bahl et al showed high breastmilk retinol levels at two months postpartum after supplementation at $18-42$ days after delivery. ${ }^{27}$ However no significant effect was noted after six months. The infants were also supplemented at immunisation visits. This combined effect improved infants' serum retinol at six months and decreased the proportion of infants with serum retinol less than $0.7 \mu \mathrm{mol} / \mathrm{l}$. Rice et al showed that supplementation of lactating Bangladeshi women benefited mothers and infants but 
did not prevent subclinical deficiency. ${ }^{28}$ Irrespective of the treatment group, over $50 \%$ of women produced milk low in vitamin A concentration $(\leqslant 1.05 \mu \mathrm{mol} / \mathrm{l})$ and the overall proportion of infants with a serum retinol content less than $0.7 \mu \mathrm{mol} / \mathrm{l}$ was $33 \%$. In another study, $56 \%$ of the breastfed infants who had received up to three doses of $50000 \mathrm{IU}$ of vitamin A still had serum retinol concentration less than 0.70 $\mu \mathrm{mol} / \mathrm{l}$ at 25 weeks of age. ${ }^{29}$ We report a much lesser number (21.92\% of the breastfed infants) at 6 months of age. In the study of Vinutha et al, at the end of three months, 69.6\% mothers in the control group had a breastmilk retinol level below $1.05 \mu \mathrm{mol} / \mathrm{l}$ against $36.1 \%$ in the experimental group. Forty five percent of infants in the control group had subclinical deficiency compared with none in the experimental group. ${ }^{21}$ In the present study $22.07 \%$ mothers in the control group had breastmilk retinol less than $0.7 \mu \mathrm{mol} / \mathrm{l}$ at the third month and $50.71 \%$ at the sixth month against $0.67 \%$ and $13.01 \%$ respectively in the test group. In the case of infants a much higher difference in their serum values was noted, $36.81 \% v 0 \%$ in the control and test groups respectively at third month. Thus, a definite impact of single megadose vitamin A supplementation to the mothers on the infants' vitamin A levels is noted continuing optimally until 4-6 months postpartum. This is the period of exclusive breastfeeding and adequate protection of the infants during this period with vitamin $\mathrm{A}$ is important to decrease morbidity and hence this is emphasised.

No case of VAD was registered in the test group, indicating a direct beneficial effect of the supplementation. Of the six cases of VAD in the control group only one showed the signs of deficiency before three months and five after three months, indicating that unsupplemented breastmilk cannot protect the infant in the later months of breastfeeding. Not only was there a significant difference in the number of episodes of acute respiratory infection $(0.34 \vee 2.55)$ between the two groups, the duration of acute respiratory infection was also significantly longer in the control group ( $2.9 v 5.6$ days). This is different from the study of Roy et al where the duration of acute respiratory infection was shorter in the supplemented infants but the incidence was comparable in both groups. ${ }^{22}$ Both the incidence and duration of diarrhoeal episodes were significantly less in the test group. A similar result was seen with febrile illnesses. Vitamin A supplementation of children is known to reduce mortality from infectious diseases. ${ }^{29}{ }^{30} \mathrm{In}$ the present study there was no infant mortality, probably because of the small sample size, and also the target group belonged to an urban population and they are relatively more health conscious and have easy access to improved health care systems. Of interest is the decreased incidence of measles in the test group, a finding consistent with previous work. ${ }^{22}$ It is probably due to improved immune status of the supplemented infants. Adequate vitamin A level is necessary for a variety of important physiological functions, including epithelial integrity and regeneration and maintenance of normal immunity. ${ }^{22}{ }^{31}$ This might explain the lesser infection rate and decreased duration of various diseases in the test group.

In a population in which breastfeeding rates are high, maternal supplementation offers several advantages. As the infant receives a daily low dose in a form that is well absorbed and utilised, it can be considered the safest way to deliver the vitamin to the newborn. Direct supplementation to the infant in the first six months of life is toxic and has shown side effects. $^{32}{ }^{33}$ Florentino et al has shown that when infants are given high dose vitamin A supplementation, transient side effect rates of $8.8 \%$ are attributable to the supplement itself. ${ }^{34}$ Secondly, maternal supplementation requires only one contact with the vitamin A delivery system, while that to the infant may require more than one. Lastly, both the mother and the infant benefit from the megadose of the vitamin. ${ }^{26}$

In conclusion, low maternal stores cannot meet the lactational demand and directly contributes to infant morbid- ity. A single oral megadose of vitamin A is readily accepted by mothers, simple to administer, and optimum for meeting lactational demand in the first six months. It also decreases morbidity and provides adequate protection to infants against VAD. Hence, such supplementation in the immediate postpartum period is emphasised along with promotion of exclusive breastfeeding to realise the maximum benefit.

\section{Authors' affiliations}

S Basu, B Sengupta, P K Roy Paladhi, Department of Pediatrics, NRS Medical College and Hospitals, Calcutta, West Bengal, India

\section{REFERENCES}

1 Beaton GH, Martorell R, Aronson KJ. Effectiveness of vitamin A supplementation in the control of young child morbidity and mortality in developing countries. United Nations ACC/SCN state of the art series; nutrition policy discussion paper No 13. Geneva, Switzerland: United Nations, 1993

2 Ghana VAST Study Team. Vitamin A supplementation in Northern Ghana: effects on clinic attendance, hospital admissions and child mortality. Lancet 1993;342:7-12.

3 Greene HL. Vitamin intakes during rapid growth. In: Heird WC, ed Nutritional needs of the six to twelve month old infants. New York: Raven Press, 1991: 251-67.

4 Newman V. Vitamin A and breastfeeding: comparison of data from developed and developing countries. San Diego: Wellstart International, 1993.

5 Sommer A, Davidson FR. Annecy Accords. Assesment and control of vitamin A deficiency: the Annecy Accords. J Nutr 2002;13219 suppl):2845S-50S.

6 Bhaskaram P. Immunobiology of mild micronutrient deficiency. Br J Nutr 2001:85(suppl 2):S75-80.

7 Duggan C, Fawzi W. Micronutrients and child health: studies in international nutrition and HIV infection. Nutr Rev 2001;59:358-69. 8 Rohler E, Wathne KO. Malnutrition and infections in children-a destructive interplay with global dimensions. Tidsskr Nor Laegeforen 2000;120:1740-5.

9 Uysal G, Guven MA, Yilmaz R, et al. Serum vitamin A levels in children with persistent diarrhoea an impact on clinical profile. J Trop Pediatr 1999:45:376-7.

10 Reyes H, Villalpando S, Perez-Cuevas R, et al. Frequency and determinants of vitamin A deficiency in children under 5 years of age with pneumonia. Arch Med Res 2002;33:180-5.

11 Chan $M$. Vitamin A and measles in Third World children. BM 1990:301:1230-1.

12 Kiolhede C, Rosales F, Caballero B, et al. Measles and low serum vitamin A values. J Pediatr 1993; 122:499-500

13 Semba RD, Bloem MW. The anaemia of vitamin A deficiency: epidemiology and pathogenesis. Eur J Clin Nutr 2002;56:271-81.

14 Reddiah VP, Gupta MC. Manual of child health and nutrition. New Delhi: Institute of Health and Nutrition, 1987.

15 Underwood BA. Maternal vitamin A status and its importance in infancy and early childhood. Am J Clin Nutr 1994;59(suppl):517S-22S.

16 Gopalan C, Rama Sastriva BV, Bala Subramanium SC. Nutrition value of Indian food. Hyderabad, India: National Institute of Nutrition, 1982.

17 Dubowitz L, Dubowitz V. Gestational age of the newborn. Reading, MA: Addison-Wesley, 1977

18 Neeld JB Jr, Pearson WN. Macro and micro methods for the determination of serum vitamin A using trifluoroacetic acid. J Nutr 1963;41:369-87

19 Rahmathulla L, Underwood BA, Thulasiraj RD, et al. Diarrhea respiratory infections and growth are not affected by a weekly low dose vitamin A supplement: a masked controlled field trial in children in southern India. Am J Clin Nutr 1991:54:568-77.

20 Mclaren DS, Frigg M. Assessment of vitamin A status. Sight and life manual on vitamin A deficiency disorders. Basel, Switzerland: Task Force Sight and Life, 1997: 36-40.

21 Vinutha B, Mehta MN, Shanbag P. Vitamin A status of pregnant women and effect of postpartum vitamin A supplementation. Indian Pediatr 2000·37:1188-93.

22 Roy SK, Islam A, Molla A, et al. Impact of a single megadose of vitamin $A$ at delivery on breastmilk of mothers and morbidity of their infants. Eur $J$ Clin Nutr 1997;51:302-7

23 Muhilal, Murdiana A, Azis I, et al. Vitamin A fortified monosodium glutamate and vitamin A status: a controlled field trial. Am J Clin Nutr 1988:48: 1265-70.

24 dePee S, West CE, Muhilal, et al. Lack of improvement in vitamin A status with increased consumption of dark-green leafy vegetables. Lancet 1995;346:75-81

25 Thanangkul O, Promkut Kaew C, Waniyapong T, et al. Comparison of the effects of a single high dose of vitamin $A$ given to mother and infant upon plasma levels of vitamin $A$ in the infant. Presented at a joint WHO/USAID meeting: The Control of Vitamin A Deficiency: Priorities for Research and Action Programmes. NUT/WP/74.114. 25-29 November Research and Action Programi
1974, Jakarta, Indonesia. 
26 Stoltzfus RJ, Hakimi M, Miller KW, et al. High dose vitamin A supplementation of breastfeeding Indonesian mothers: effects on the vitamin A status of mother and infant. J Nutr 1993;123:666-75.

27 Bahl R, Bhandari N, Wahed MA, et al. The WHO/CHD

Immunization-linked' Vitamin A Group. Vitamin A supplementation of women postpartum alters breastmilk retinol and infant vitamin $A$ status. $J$ women postpartum alters

28 Rice AL, Stoltzfus RJ, de Fransisco A, et al. Maternal vitamin A or beta-carotene supplementation in lactating Bangladeshi women benefits mothers and infants but does not prevent subclinical deficiency. J Nutr 1999;129:356-65.

29 Mahalanabis D, Rahman MM, Wahed MA, et al. Vitamin A megadoses during early infancy on serum retinol concentration and acute side effects and residual effects on 6 month follow up. Nutr Res 1997;17:649-59.
30 Humphrey JH, Agoestina T, Wu L, et al. Impact of neonatal vitamin A supplementation on infant morbidity and mortality. J Pediatr 1996; 128:489-96

31 Bhaskaram P. Micronutrient malnutrition, infection and immunity: an overview. Nutr Rev 2002;60(5 pt 2):S40-5.

32 de Francisco A, Chakraborty J, Chowdhury HR, et al. Acute toxicity of vitamin A given with vaccines in infancy. Lancet 1993;342:526-7.

33 Gopalan L. Acute toxicity of vitamin A in infancy. Bulletin of the Nutrition Foundation 1994;15:1.

34 Florentino RF, Tanchoco CC, Ramos AC, et al. Tolerance of preschoolers to two dosage strengths of vitamin A preparation. Am J Clin Nutr 1990;52:694-700.

\section{IMAGES IN MEDICINE}

\section{An informative tattoo}

28 year old man with type 1 diabetes had this tattoo inscribed a few weeks after hypoglycaemin and Has particularly concerned about this being unrecognised by others. He decided to have a tattoo rather than carry a Medic Alert bracelet.

Hypoglycaemia is the most common complication of insulin therapy and one that patients on insulin fear the most. Neuroglycopenic symptoms due to hypoglycaemia are not infrequently mistaken for alcohol intoxication. The frequency with which this occurs suggests that such accounts may be more than just anecdotal.

S Nag, A McCulloch

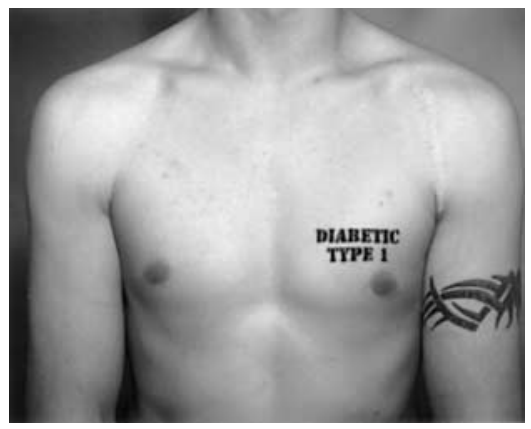

(Published with patient's permission.)

Bishop Auck Road, Bishop Auckland DL14 6AD, UK; s.nag@btopenworld.com 